\title{
Endothelial cells and endothelin-1 promote the odontogenic differentiation of dental pulp stem cells
}

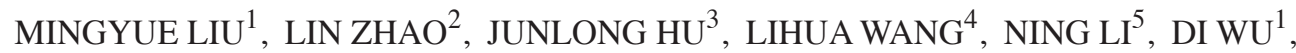 \\ $\mathrm{XIN} \mathrm{SHI}^{1}$, MENGTONG YUAN $^{1}$, WEIPING HU ${ }^{1}$ and XIAOFENG WANG ${ }^{4}$ \\ ${ }^{1}$ Department of Prosthodontics, The Second Affiliated Hospital of Harbin Medical University, Harbin, Heilongjiang 150086; \\ ${ }^{2}$ Department of Stomatology, Dezhou People's Hospital, Dezhou, Shandong 253000; ${ }^{3}$ Department of Neurosurgery, \\ The First Affiliated Hospital of Harbin Medical University; Departments of ${ }^{4}$ Stomatology and ${ }^{5}$ Cardiology, \\ The Second Affiliated Hospital of Harbin Medical University, Harbin, Heilongjiang 150086, P.R. China
}

Received November 8, 2017; Accepted April 13, 2018

DOI: $10.3892 / \mathrm{mmr} .2018 .9033$

\begin{abstract}
It has been established that dental pulp stem cells (DPSCs) serve an important role in the restoration and regeneration of dental tissues. DPSCs are present in blood vessels and also exist in the vessel microenvironment in vivo and have a close association with endothelial cells (ECs). The present study aimed to evaluate the influence of ECs and their secretory product endothelin-1 (ET-1) on the differentiation of DPSCs. In the present study, cells were divided into four groups: i) a DPSC-only control group; ii) a DPSC with ET-1 administration group; iii) a DPSC and human umbilical vein endothelial cell (HUVEC) direct co-culture group; and iv) a DPSC and HUVEC indirect co-culture group using a Transwell system. Reverse transcription-quantitative polymerase chain reaction was used to detect the expression of the odontoblastic differentiation-associated genes, including dentin sialoprotein (DSP) and dentin matrix acidic phosphoprotein 1 (DMP-1) at days 4, 7, 14 and 21. Alizarin Red S staining, immunofluorescence and western blot analyses were also conducted to assess the differentiation of the DPSCs in each group. The highest expression levels of odontoblastic differentiation-associated genes were observed on day 7 and in the two co-culture groups were increased compared with the DPSC-only and DPSC + ET-1 culture groups at all four time points. However, expression levels in the DPSC + ET-1 group were not downregulated as notably as in the co-culture groups
\end{abstract}

Correspondence to: Dr Xiaofeng Wang, Department of Stomatology, The Second Affiliated Hospital of Harbin Medical University, 246 Xuefu Road, Harbin, Heilongjiang 150086, P.R. China

E-mail: wxf_hrbmu@163.com

Dr Weiping $\mathrm{Hu}$, Department of Prosthodontics, The Second Affiliated Hospital of Harbin Medical University, 246 Xuefu Road, Harbin, Heilongjiang 150086, P.R. China

E-mail: hwp1963@126.com

Key words: human umbilical vein endothelial cells, endothelin-1, dental pulp stem cells, odontoblastic differentiation, co-culture on days 14 and 21 . The Transwell group exhibited the greatest ability for odontoblastic differentiation compared with the other groups according to staining with Alizarin Red S, immunofluorescence and western blot analysis results. According to the results of the present study, the culture solution with HUVECs affected the differentiation of DPSCs. In addition, ET-1 may promote the odontoblastic differentiation of DPSCs.

\section{Introduction}

Researchers have paid an increasing amount of attention to the applications of dental pulp stem cells (DPSCs) in the field of dental regenerative medicine. DPSCs can differentiate into dental tissue and bone tissue in vitro (1). Additionally, endothelial cells (ECs) can secrete a series of bioactive substances, including endothelin-1 (ET-1) and insulin-like growth factor (IGF). Shi and Gronthos (2) reported that DPSCs were present in the micrangium region of dental pulp. Cell staining also revealed that STRO-1 (a marker of mesenchymal cells), cluster of differentiation (CD)146 (a marker of endothelial cells) and $\alpha$-smooth-muscle actin (a marker of pericytes) were positively expressed on the surface of perivascular cells (2). Notably, the elevated levels of CD146 expression suggested a perivascular origin of DPSCs. The migration of pre-odontoblasts to blood vessels may be due to the degradation reaction of dentin (3). In turn, ECs may regulate the development of dentine/pulp tissue. Additionally, ECs may control the proliferation of cells by maintaining the stabilization of blood vessels and secreting relevant molecules, and, therefore ECs may be considered to be a novel resource for tissue regeneration (3). Mathieu et al (4) demonstrated that dental pulp was a type of vascularized tissue, which may stimulate ECs to secrete chemokines and signaling molecules upon infection. Subsequently, Mathieu et al (4) observed that the inflammation began to promote the secretion of inflammatory factors and adhesion molecules, which DPSCs require to accelerate the repair processes within the tissue. Factors including fibroblast growth factor 2 (FGF-2), secreted by ECs, participated in angiogenesis and DPSC division (5).

The aforementioned research demonstrated that there may be an interaction between ECs and DPSCs. Dissanayaka et al (6) 
directly co-cultured ECs and DPSCs and reported that ECs may regulate the odontoblastic differentiation of DPSCs. Additionally, DPSCs may induce ECs to generate a vascular-like tissue structure (7). It has been suggested that this promotion of differentiation and proliferation may be due to ET-1 and IGF $(8,9)$, which are secreted by ECs; however, the direct co-culture with these two cell types may also be the reason for the promotion of these processes $(3,10,11)$. Sueyama et al (12) implanted mesenchymal stem cells (MSCs) with endothelial cells (ECs), and observed accelerated pulp tissue regeneration/healing and induction of dentin bridge formation in a rat model of molar coronal pulp regeneration.

ETs were originally identified by Yanagisawa in 1988 (13). The main role of ETs is to maintain vascular homeostasis under physiological conditions, as well as during nociception and periods of local inflammation (14-16). There are three different subtypes of ETs, namely ET-1, ET-2, and ET-3. ET-1 is the most common type observed in humans (17). ET-1 is a type of bioactive peptide composed of 21 amino acid residues, and may be extracted from aortic endothelial cells; ET-1 affects the proliferation and differentiation of MSCs, and preosteoblasts, as reported by Sin et al (18). ET-1 can also maintain vascular tension and stability in the cardiovascular system (19). Furthermore, it serves a significant role in the development of diseases, including hypertension and atherosclerosis (20). In the culture of rat ophthalmic arteries, ET-1 can mediate vasoconstriction (21). There is substantial evidence that, in numerous pathophysiologies associated with endothelial dysfunction, ET-1 may release potent vasoconstrictors and sustain elevated vascular tone; however, there is considerably less data to support the role of ET-1 in the regulation of vascular tone under physiological conditions (22-24). In addition, ET-1 also serves a role in osteogenesis and bone remodeling. Sin et al (18) indicated that ET-1 may induce the differentiation of osteoblasts via the membrane protein ankyrin 43. In addition, ET-1 may enhance the mRNA expression of osteopontin and osteocalcin, and stimulate the release of alkaline phosphatase and secretion of collagenase type I (25); however, compared with its expression in osteoblasts, ET-1 can be detected on the cell membrane and in the cytoplasm of osteoclasts by immunostaining (26). This indicated that osteoclasts may be a target cell affected by ET-1. Accordingly, ET-1 may accelerate the resorption of bone by endothelin type A (ET A) receptor (18). Therefore, ECs and ET-1 may regulate osteogenesis, remodeling and bone resorption by controlling the activity of osteoblasts and osteoclasts. These findings present the advances in bone research regarding ET-1 function. Several studies have investigated odontogenesis with regards to the potential involvement of ET-1. Warner (27) revealed that ET-1 affected the concentration of calcium in contractile fiber cells via the ET A receptor. Additionally, a high concentration of ET-1 during the process of dental morphogenesis can be observed (28). It has been suggested that fibronectin (FN) serves an important role in the formation of the tooth root. A previous study demonstrated that ET-1 may induce the production of extracellular matrix protein and FN (29). Therefore, the additional function of ET-1 may be to promote the proliferation and differentiation of cells in the tooth root. Injection of ET into the dental pulp of dogs can cause vasoconstriction and decreased blood circulation (30), demonstrating that receptors for ET also exist in the dental pulp. When treated with ET-1, there is a constant release of $\mathrm{Ca}^{2+}$ from DPSCs (3). Preconditioning of MSCs with ET-1 exhibits strong cytoprotective effects via the activation of survival signaling molecules and trophic factors (31).

Despite the aforementioned findings, it is evident that the mechanism of ECs and ET-1 in histogenesis and tissue formation requires further investigation. Therefore, the present study aimed to investigate the effect of human umbilical vein endothelial cells (HUVECs) and ET-1 on DPSC differentiation in vitro.

\section{Materials and methods}

Primary pulp cell cultures. Human pulp cells were prepared from immature third molars at the $2 / 3$ root formation stage by the explant outgrowth method (32). The teeth were obtained from at least three different donors for each experiment $(n=12$; four molars per donor; age, 18-25 years; 1:1 male to female). Surgeries were performed at the Oral and Maxillofacial Surgery Department of the Second Affiliated Hospital of Harbin Medical University (Harbin, China). HUVECs were provided by the Research and Test Center of the Second Affiliated Hospital of Harbin Medical University (Harbin, China). The present study was approved by the Institutional Ethics Committee of the Second Affiliated Hospital of Harbin Medical University (Harbin, China). Written informed consent was obtained from all patients. The basic cell culture medium used consisted of Dulbecco's modified Eagle's medium/F-12 (Hyclone; GE Healthcare Life Sciences, Logan, UT, USA), supplemented with $15 \%$ fetal bovine serum (Biological Industries, Kibbutz Beit Haemek, Israel) and $1 \%$ penicillin and $1 \%$ streptomycin.

Cell groups and culture conditions. Cells were cultured in a $37^{\circ} \mathrm{C}$ incubator with $5 \% \mathrm{CO}_{2}$. Cells (all $8 \times 10^{4}$ ) were divided into four groups: i) a DPSC-only control group; ii) a DPSC with ET-1 $\left(10^{-8} \mathrm{M}\right)$ administration group; iii) a DPSC and HUVEC direct co-culture group (DPSCs:HUVECs, 5:1); and iv) a DPSC and HUVEC indirect co-culture group using a Transwell system, in which HUVECs were inoculated into the chamber at a density of $2 \times 10^{4}$ cells. The odontoblastic differentiation culture medium consisted of basic cell culture medium with $10 \mathrm{nmol} / \mathrm{l}$ dexamethasone, $5 \mathrm{mmol} / 1 \beta$-glycerophosphate and $50 \mathrm{mg} / \mathrm{ml}$ vitamin-C-phosphate.

Induction of DPSC differentiation into adipocytes, chondroblasts and osteoblasts. Cells were cultured to the third generation in a $37^{\circ} \mathrm{C}$ incubator with $5 \% \mathrm{CO}_{2}$. All groups of cells were plated at a density of $5 \times 10^{4}$ per well. To induce DPSCs to differentiate into adipocytes, the culture medium was supplemented with $0.5 \mu \mathrm{M}$ isobutylmethylxanthine, $50 \mu \mathrm{M}$ indomethacin and $0.5 \mu \mathrm{M}$ dexamethasone for 3 weeks. The adipogenic cultures were fixed in $4 \%$ paraformaldehyde for $30 \mathrm{~min}$ at room temperature and stained with fresh Oil Red O solution for $1 \mathrm{~h}$ at room temperature. The chondroblast induction medium consisted of $1 \mu \mathrm{M}$ dexamethasone, $37.5 \mu \mathrm{g} / \mathrm{ml}$ vitamin-C-phosphate, $1 \mathrm{mM}$ sodium pyruvate, $10 \mathrm{ng} / \mathrm{ml}$ transforming growth factor- $\beta$ (TGF- $\beta$ ), $1 \mathrm{ng} / \mathrm{ml} \beta$-FGF, $1 \mathrm{X}$ Insulin-Transferrin-Selenium premix (Sigma-Aldrich; Merck KGaA, Darmstadt, Germany), in 
which DPSCs were maintained for 3 weeks. The chondrogenic cultures were fixed in $4 \%$ paraformaldehyde for $30 \mathrm{~min}$ at room temperature and stained with toluidine blue for $30 \mathrm{~min}$ in a $37^{\circ} \mathrm{C}$ incubator. The osteoblast induction medium contained $10 \mathrm{nmol} / 1 \mathrm{dexamethasone,} 5 \mathrm{mmol} / \mathrm{l} \beta$-glycerophosphate and $50 \mathrm{mg} / \mathrm{ml}$ vitamin-C-phosphate, in which DPSCs were cultured for 3 weeks. The osteoblast cultures were fixed in $95 \%$ ethanol for $30 \mathrm{~min}$ at $37^{\circ} \mathrm{C}$ and stained with Alizarin Red $\mathrm{S}$ for $30 \mathrm{~min}$ in a $37^{\circ} \mathrm{C}$ incubator. Cells were observed under a light microscope (original magnification, $\mathrm{x} 40$ ).

Cytotoxicity (MTT) assay. This assay employed two control groups: i) a DPSC-only culture group; and ii) a HUVEC-only culture group. Two experimental groups were also employed: i) a DPSC plus $10^{-8} \mathrm{M}$ ET-1 group; and ii) a DPSC and HUVEC (5:1) direct co-culture group. The present study employed 96-well plates with $1 \times 10^{3}$ cells/well. Dimethyl sulfoxide $(200 \mu \mathrm{l})$ was added to dissolve the formazan crystals. The absorbance was measured with an ELISA reader (Thermo Fisher Scientific, Inc., Waltham, MA, USA) at a wavelength of $490 \mathrm{~nm}$. The cell viability ratio was calculated using the following formula:

Inhibitory ratio $(\%)=$ optical density $(\mathrm{OD})$ control - OD treated $) /(\mathrm{OD}$ control $)] \times 100$.

Mineralization induction and quantification. This assay used four groups: i) a DPSC-only control group; ii) a DPSC with ET-1 administration group; iii) a DPSC and HUVEC direct co-culture group; and iv) a DPSC and HUVEC indirect co-culture group using a Transwell system. Cells $\left(5 \times 10^{4} /\right.$ well) were seeded onto 6 -well plates and cultured for 21 days. Calcium accumulation was detected by fixing the cultures with $95 \%$ ethanol for $30 \mathrm{~min}$ at $37^{\circ} \mathrm{C}$, followed by staining with $0.1 \%$ Alizarin Red S (Sigma-Aldrich; Merck KGaA) at $37^{\circ} \mathrm{C}$ for $30 \mathrm{~min}$. To quantify matrix mineralization, the cultures stained with Alizarin Red $\mathrm{S}$ were incubated with $100 \mathrm{mM}$ cetylpyridinium chloride for $1 \mathrm{~h}$ at $37^{\circ} \mathrm{C}$ to solubilize and release calcium-bound Alizarin Red into solution. Subsequently, $200 \mathrm{ml}$ aliquots were transferred onto a 96-well plate and the OD of the solution was measured at a wavelength of $570 \mathrm{~nm}$ using a microplate reader (Tanon Science \& Technology Co., Ltd., Shanghai, China). Mineralized nodule formation was represented as $\mathrm{OD} / \mu \mathrm{g}$ of total cellular protein, determined using a Bradford protein assay. Experiments were performed in triplicate wells and were repeated at least three times.

Reverse transcription-quantitative polymerase chain reaction (RT-qPCR) analysis. The four groups of cells were cultured in mineralized solution (in a $37^{\circ} \mathrm{C}$ incubator with $5 \% \mathrm{CO}_{2}$ ) and total RNA was extracted on days 4, 7, 14 and 21 to detect the gene expression of dentin sialoprotein (DSP) and dentin matrix acidic phosphoprotein 1 (DMP-1). Total RNA was extracted using TRIzol reagent (Invitrogen; Thermo Fisher Scientific, Inc.) according to the manufacturer's instructions. Subsequently, the RNA was converted to cDNA using a Transcriptor First Strand cDNA Synthesis kit (Roche Diagnostics $\mathrm{GmbH}$, Mannheim, Germany) using the following temperature protocol: $50^{\circ} \mathrm{C}$ for $60 \mathrm{~min}, 85^{\circ} \mathrm{C}$ for $5 \mathrm{~min}$ and $4^{\circ} \mathrm{C}$ for $10 \mathrm{~min}$. The expression levels of the genes were quantified using FastStart Universal SYBR-Green Master Rox mix
(Roche Diagnostics GmbH). The PCR thermocycling conditions were as follows: $95^{\circ} \mathrm{C}$ for $2 \mathrm{~min}$, followed by 40 cycles of $95^{\circ} \mathrm{C}$ for $15 \mathrm{sec}$ and $60^{\circ} \mathrm{C}$ for $30 \mathrm{sec}$. PCR results were normalized against the reference gene GAPDH to correct for non-specific experimental variation. The method of $\Delta \Delta \mathrm{Cq}$ was used to determine the relative quantity of mRNA expression in samples, and fold change was determined as $2^{-\Delta \Delta \mathrm{Cq}}$ (33). The following primers were used: DSP forward, 5'-TTTCCGCTT GTCATCATCTCC-3' and reverse, 5'-GGTGTCCTGGCA CTACTGCAT; DMP-1 forward, 5'-AAAATTCTTGTGAAC TACGGAGG-3' and reverse, 5'-GAGCACAGGATAATCCC CAA-3'; GAPDH forward, 5'-GACAACTCCCTCAAGATT GTCAG-3' and reverse, 5'-ATGGCATGGACTGTGGTCAT GAG-3'.

Immunofluorescence staining. The four groups of cells were cultured in 6 -well plates $\left(5 \times 10^{4}\right.$ cells/well) for 2 weeks prior to immunostaining. Cells were fixed in $4 \%$ paraformaldehyde for $30 \mathrm{~min}$ at room temperature and permeabilized in $0.2 \%$ Triton X-100 for $15 \mathrm{~min}$. Cells were blocked in 3\% bovine serum albumin (BSA, Sigma-Aldrich; Merck KGaA) for $1 \mathrm{~h}$ at room temperature. Odontoblasts were detected using a specific antibody against DSP at $4^{\circ} \mathrm{C}$ overnight (cat. no. sc-33586; 1:50; Santa Cruz Biotechnology, Inc., Dallas, TX, USA). Cells were subsequently washed 3 times in PBS with $0.3 \%$ Tween for $5 \mathrm{~min}$ each wash, and incubated for $1 \mathrm{~h}$ at room temperature with Alexa Fluor-conjugated goat anti-rabbit IgG (cat. no. E031320-01; 1:500; EarthOx Life Sciences, Millbrae, CA, USA). Cell nuclei were subsequently stained with DAPI (cat. no. C1005; Beyotime Institute of Biotechnology, Haimen, China) for $5 \mathrm{~min}$ at room temperature. Immunofluorescence was detected using a fluorescence microscope (magnification, x20; DMI14000B; Leica Microsystems GmbH, Wetzlar, Germany).

Western blot analysis. Total protein was extracted from the four groups of cells on day 7 using cell lysis buffer $[20 \mathrm{mM}$ Tris ( $\mathrm{pH} 7.5), 150 \mathrm{mM} \mathrm{NaCl}, 1 \%$ Triton X-100, $2.5 \mathrm{mM}$ sodium pyrophosphate, $1 \mathrm{mM}$ EDTA, $1 \% \mathrm{Na} 3 \mathrm{CO} 4,0.5 \mu \mathrm{g} / \mathrm{ml}$ leupeptin, $1 \mathrm{mM}$ phenylmethanesulfonyl fluoride]. The lysates were collected by scraping from the plates and subsequently centrifuged at $15,000 \mathrm{x} \mathrm{g}$ at $4^{\circ} \mathrm{C}$ for $5 \mathrm{~min}$. Protein concentration was quantified using a bicinchoninic acid assay. Total protein samples $(20 \mu \mathrm{g} /$ lane $)$ were separated by $12 \%$ SDS-PAGE and transferred onto polyvinylidene fluoride membranes. Membranes were blocked in $1 \%$ BSA with $0.05 \%$ Tween-20 at room temperature for $2 \mathrm{~h}$. Membranes were incubated overnight at $4^{\circ} \mathrm{C}$ with the following antibodies: Rabbit anti-human DSP (cat. no sc-33586; 1:200; Santa Cruz Biotechnology, Inc.), and $\beta$-actin (cat. no. TA346894; 1:500, Zhongshan Goldenbridge, Beijing, China). The secondary antibody used were horseradish peroxidase-conjugated AffiniPure goat anti-rabbit IgG (cat. no. E030120-01; 1:10,000; EarthOx Life Sciences, Millbrae, CA, USA) and goat anti-mouse IgG (cat. no. E030110-01; 1:10,000; EarthOx Life Sciences). Bands were visualized using an enhanced chemiluminescence western blot kit (CWBIO, Beijing, China) and a Tanon 1000 digital image gel analytical system (Tanon Science \& Technology Co., Ltd., Shanghai, China) was used for photography and quantification. 

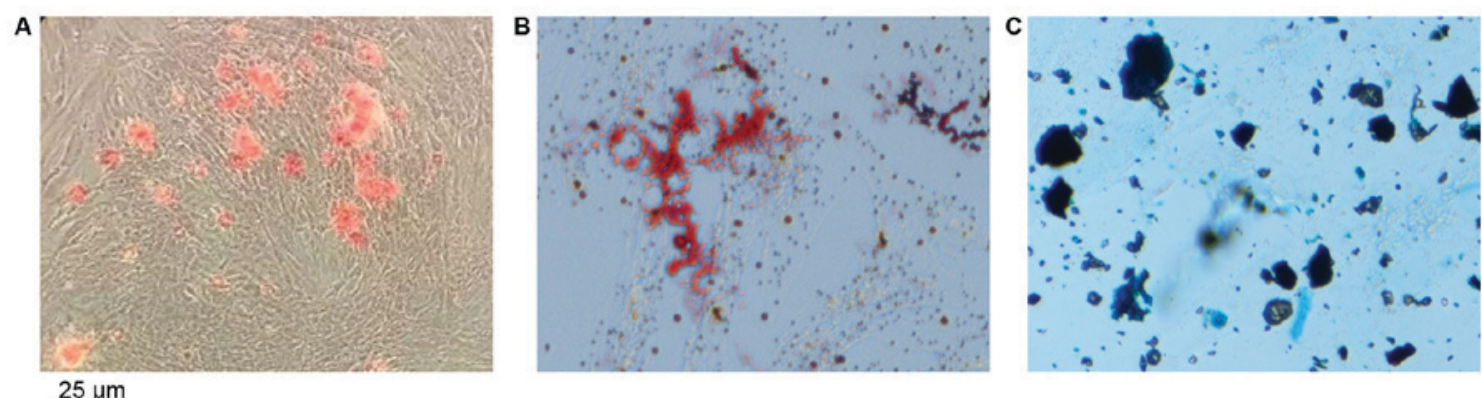

Figure 1. Multilineage differentiation capacity of dental pulp stem cells (original magnification, $\mathrm{x} 40$ ). (A) Osteogenic differentiation was indicated by the deposition of mineralized matrix, detected by Alizarin Red S staining. (B) Adipogenic differentiation was indicated by the accumulation of neutral lipid vacuoles, detected with Oil Red O staining. (C) Chondroblasts were detected by toluidine blue staining. Scale bar, $25 \mu \mathrm{m}$.

Statistical analysis. All values are expressed as the mean \pm standard error of the mean. Statistical analysis was performed by using one-way analysis of variance followed by Bonferroni multiple comparisons using SPSS 13.0 software (SPSS, Inc., Chicago, IL, USA). P<0.05 was considered to indicate a statistically significant difference.

\section{Results}

Characterization of the immunophenotype and differentiation potential of DPSCs in vitro. The presence of mineralized nodules demonstrated successful osteogenic induction of DPSCs (Fig. 1A). As presented in Fig. 1B, the formation of neutral lipid vacuoles also indicated the adipogenic potential of the DPSCs. Furthermore, the DPSCs were observed to differentiate into chondroblasts following induction (Fig. 1C).

Cytotoxicity (MTT) assay. The data for the HUVEC-only group exhibited the highest level of cell proliferation among the four groups. DPSC and HUVEC direct co-culture group revealed that cells underwent notable proliferation, and proliferation was significantly increased at day 10 and 14 , compared with the DPSC + ET-1 and DPSC only groups. The DPSC + ET-1 and DPSC only groups exhibited almost linear increases from day 3 to day 14 (Fig. 2).

Mineralization assay. The cells adopted an osteoblast-like polygonal morphology after 21 days of culture. Following the induction of mineralization, white mottled crystals appeared, which became red mineralized nodules following staining with Alizarin Red S. As presented in Fig. 3A, the mineralized nodules appeared to be smaller and less abundant within the DPSC-only group compared with in the ET-1 treatment (Fig. 3B), Transwell (Fig. 3C) and co-culture group (Fig. 3D). Nodule formation was observed to be significantly higher in the DPSC + ET-1, Transwell and DPSC and HUVEC groups compared with in the control. The Transwell group generated the highest number of nodules of all four groups (Fig. 3E).

RT-qPCR analysis. Odontogenesis-associated genes were expressed in all four groups. DSP and DMP-1 were expressed weakly in the DPSC-only culture group compared with in the other three groups. Over the experimental period, the expression of DSP and DMP-1 was highest on day 7, with successively lower levels detected from day 14 to day 21 in all four groups.

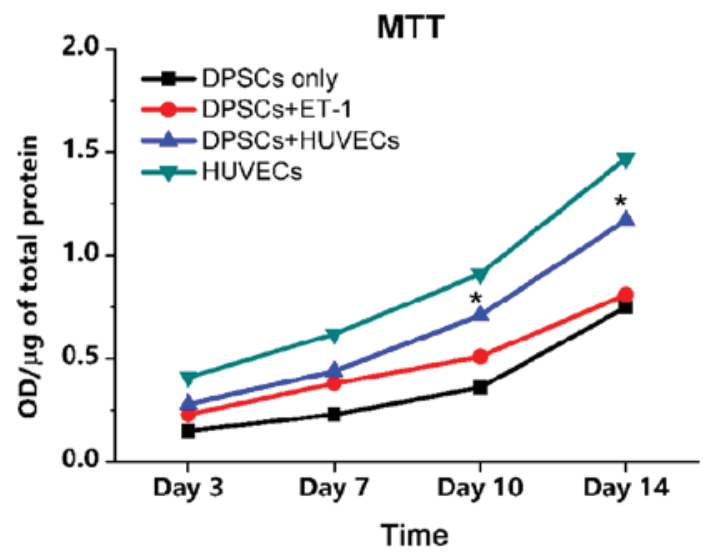

Figure 2. MTT assay. The DPSC and HUVEC co-culture group underwent marked proliferation compared with in the DPSC+ET-1, DPSC-only and HUVEC-only groups. ${ }^{*} \mathrm{P}<0.05$ vs. DPSC + ET -1 and DPSC only groups. DPSC, dental pulp stem cell; ET-1, endothelin-1; HUVEC, human umbilical vein endothelial cell.

The expression levels of the two genes were highest in the DPSC + HUVEC direct co-culture group on day 4, while being second highest in the Transwell culture group. The two genes were expressed at the highest level on days 7 and 14 in the Transwell culture group, with lower levels detected in the direct co-culture group, the DPSC + ET-1 group and the DPSC-only group, over this period. The expression of the odontogenesis-associated genes was notably downregulated on day 21 in the direct co-culture and Transwell culture groups, but remained significantly higher compared with the DPSC + ET-1 group (Fig. 4A).

Immunofluorescence staining. Immunofluorescence staining revealed that all four groups expressed DSP after 2 weeks of culture; however, expression was weak in the DPSC-only group (Fig. 4B).

Protein expression during the process of DPSC differentiation. The western blot assay demonstrated that compared with the DPSC-only group, the expression levels of DSP protein were significantly increased in the DPSC + ET-1 group, the direct co-culture group and the Transwell culture group. The expression of DSP in the Transwell group was significantly higher than the direct co-culture group. DSP expression in the DPSC + ET-1 group was lower than the above two groups (Fig. 5). 

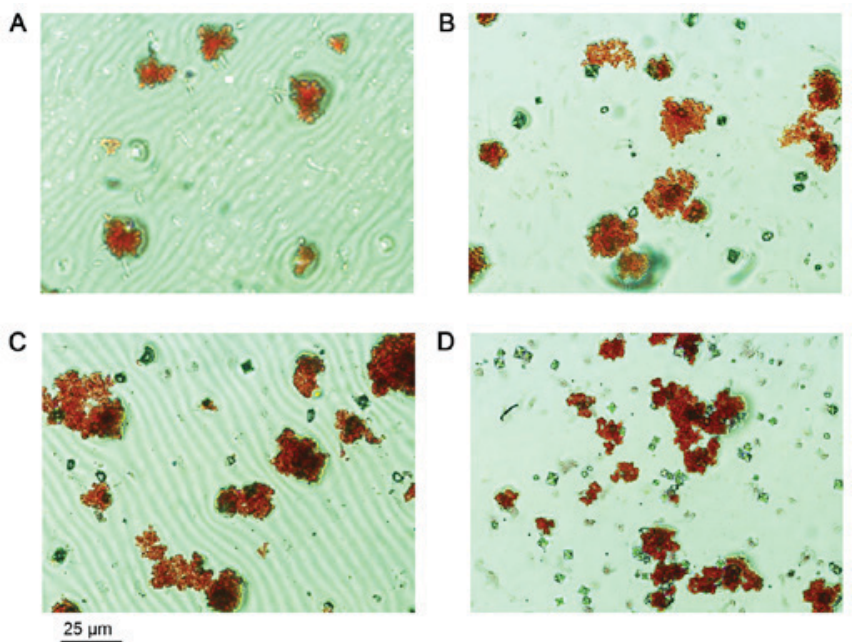

E

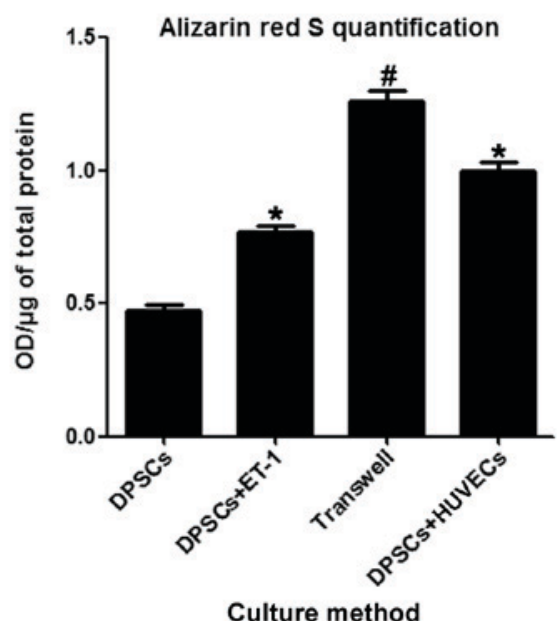

Figure 3. Cell morphology and mineralized nodules of human dental pulp cells maintained in mineralized medium for 21 days. Mineralized nodules were stained by Alizarin Red S (original magnification, x40). (A) DPSC-only group; (B) DPSC + ET-1 group; (C) DPSC and HUVEC indirect co-culture group using a Transwell system and (D) DPSC and HUVEC direct co-culture group. (E) Quantitation of Alizarin Red S staining at day 21. Data are presented as the mean $\mathrm{OD} / \mu \mathrm{g}$ of total protein \pm standard deviation $(\mathrm{n}=6)$ ${ }^{*} \mathrm{P}<0.05$ vs. DPSC-only group. ${ }^{\#} \mathrm{P}<0.05$ vs. other three groups. DPSC, dental pulp stem cell; ET-1, endothelin-1; HUVEC, human umbilical vein endothelial cell; OD, optical density.

\section{Discussion}

It is widely acknowledged that further study on DPSCs may have a positive impact on dental tissue engineering and tooth regeneration. DPSCs present in their perivascular niche and ECs present on the surface of blood vessels interact directly or indirectly within the tumor microenvironment. When caries or dental-trauma occurs, the direct cell-cell contact may affect the reparation and regeneration of dental tissue to a certain extent; however, whether the direct contact or interaction between these two cell types promotes the secretion of cytokines requires further investigation. The present study divided DPSCs and HUVECs into various co-culture groups, in order to compare the different factors that may impact on the differentiation of dental tissue (2,34-38). Researchers have suggested that HUVECs notably affect the function of DPSCs, which is in accordance with the findings of other studies (9,34-36,38-40). Saleh et al (9) reported that HUVECs may promote the survival, proliferation and aggregation of bone marrow mesenchymal stem cells. Dissanayaka et al (6) directly co-cultured HUVECs and DPSCs, and revealed that HUVECs promoted the proliferation and differentiation of DPSCs. Therefore, four groups were designed in the present study, namely the Transwell co-culture, direct co-culture, DPSC + ET-1 and DPSC-only groups. The Transwell method enabled the cytokines secreted from HUVECs to migrate via a semipermeable membrane into the culture below, while preventing HUVECs from entering the DPSC culture. The detection of odontogenesis-associated genes indicated that the two co-culture groups expressed the genes at increased levels compared with the DPSC-only group on days 4, 7, 14 and 21. The expression levels of the genes declined from peak levels on day 7 . The results of the present study revealed that the process of co-culture may promote the differentiation of DPSCs, and that this capability increased on day 7 and then decreased throughout further cell culture. The results on day 4 indicated elevated expression levels of the odontogenic markers in the direct co-culture group compared with in the Transwell group, which may have been due to the direct contact of the two cell types initially promoting the differentiation of the DPSCs. This promotion may be unassociated with cytokine secretion. The lower expression levels in the Transwell group on day 4 may support this hypothesis; however, the DPSC + ET-1 and the DPSC-only groups expressed lower levels of the odontogenic markers compared with the two co-culture groups on day 4. This result demonstrated that HUVECs may be advantageous to DPSC differentiation and the cytokines secreted by the HUVECs may have promoted the differentiation of the DPSCs. The effect on differentiation induced by ET-1 alone was not as strong as that in the co-culture groups. The results of the present study indicated that ET-1 may be an effective factor promoting the differentiation of DPSCs. This is in accordance with research on developing rat teeth (28); however, there may be other cytokines that accompany ET-1 in promoting the differentiation of DPSCs, as the results of the co-culture groups demonstrated in the present study. The results on day 7 and day 14 indicating increased odontogenic gene expression in the Transwell group may be due to the duration of culture. The direct contact between the two cell types may serve a stimulatory role; however, the promotional function may have been limited over time as the interaction between the two cells was unfavorable to the secretion of HUVECs, thereby affecting the differentiation of the DPSCs. Odontogenic gene expression in the Transwell group increased until day 7, after which the levels were downregulated, which was consistent with the results of our previous research (32). Our previous research demonstrated that during the mineralization process of DPSCs, the ability for odontogenesis decreased over time (32). Alternatively, the effect on differentiation may be due to increasing cell density. The measurements on day 21 in the present study revealed that the two co-culture groups exhibited downregulated expression levels of the odontogenic markers compared with in the other groups; however, the differences between the two groups were not significant.

There are two potential reasons for the downregulated expression, including that the density of the cells may have affected the differentiation. Additionally, the extended duration of direct contact may have inhibited differentiation; however, 
A

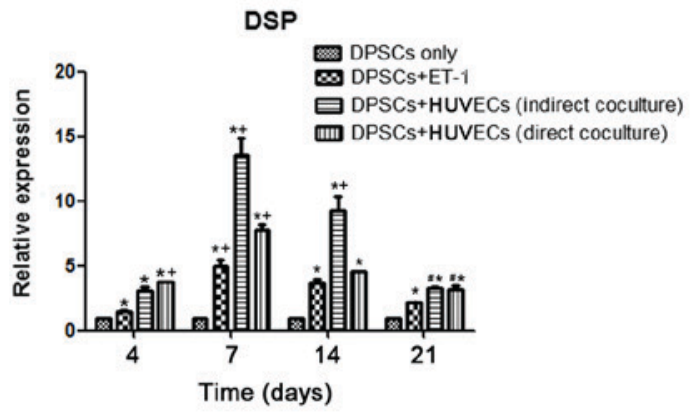

B a

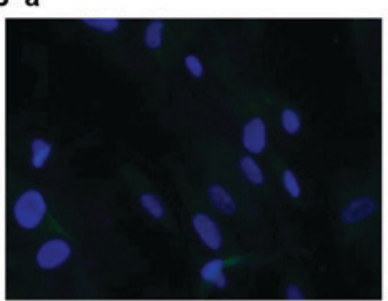

b

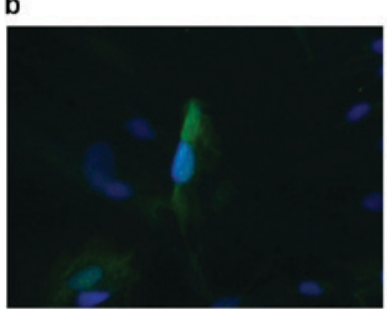

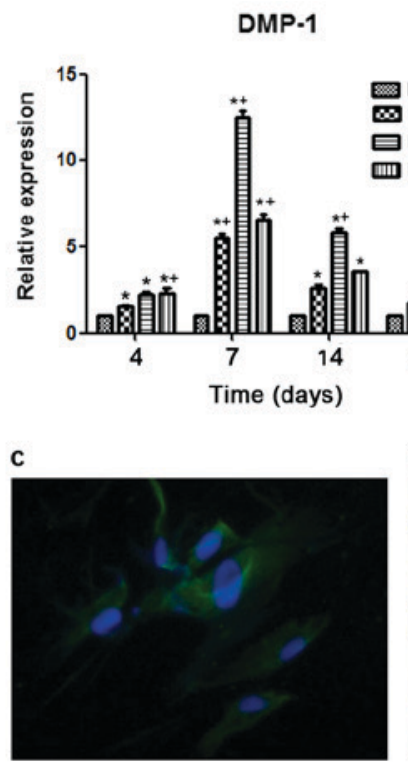

Figure 4. (A) Relative expression levels of odontogenic markers at different time points detected by reverse transcription-quantitative polymerase chain reaction. Values were normalized to GAPDH expression. ${ }^{*} \mathrm{P}<0.05$ vs. DPSC-only group at the corresponding time point. ${ }^{+} \mathrm{P}<0.05$ vs. the corresponding group at different time points. ${ }^{*} \mathrm{P}<0.05$ vs. DPSCs + ET-1 group at day 21. (B) Immunofluorescence detection of DSP protein expression. (B-a) DPSC-only group; (B-b) DPSC + ET-1 group; (B-c) DPSC + HUVEC indirect co-culture group using a Transwell system and (B-d) DPSC + HUVEC direct co-culture group. DSP expression is indicated by green staining; nuclei were counter-stained with DAPI (blue). DPSC, dental pulp stem cell; DMP-1, dentin matrix acidic phosphoprotein 1; DSP, dentin sialoprotein; ET-1, endothelin-1; HUVEC, HUVEC, human umbilical vein endothelial cell.

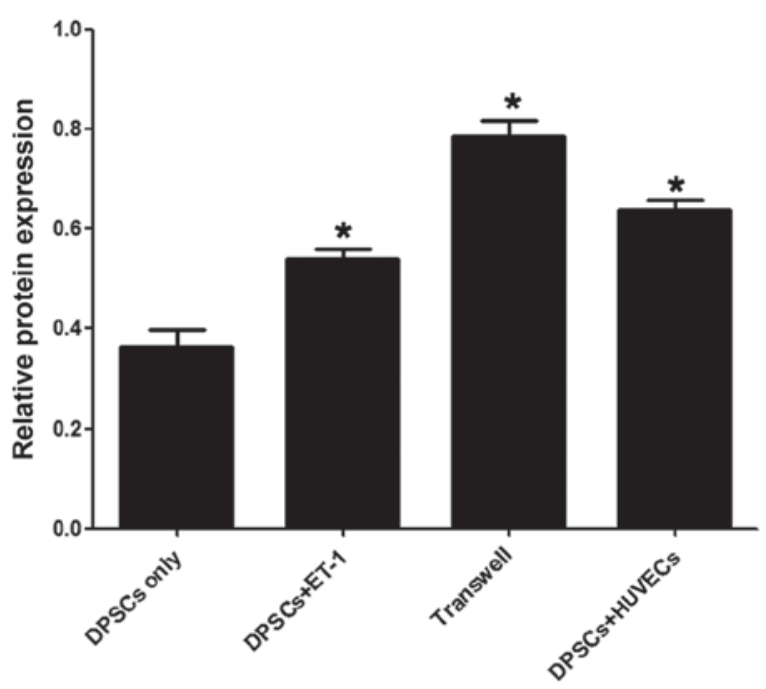

Culture method

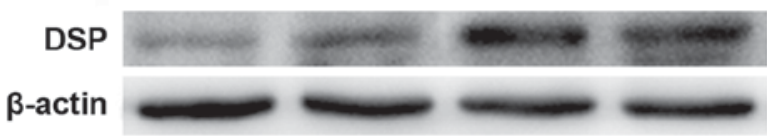

Figure 5. DSP expression detected by western blotting. The results were presented as the value calculated from three different cell samples and each same cell sample was assayed in triplicate ${ }^{*} \mathrm{P}<0.05$ vs. DPSC-only group. DPSC, dental pulp stem cell; DSP, dentin sialoprotein; ET-1, endothelin-1; HUVEC, human umbilical vein endothelial cell.

it is evident that there were numerous potential factors that may have exerted an effect on the promotional effects of the HUVECs. For instance, HUVECs can secrete insulin growth factor-1, ET-1, FGF, platelet-derived growth factor, TGF- $\beta$ and bone morphogenic protein-2 $(9,10)$, which may promote the proliferation and aggregation of progenitor cells. Except for ET-1, researchers have focused on these factors and investigation has revealed that IGF-1 may promote the proliferation and differentiation of DPSCs (41). This stimulatory effect was associated with the activation of the mechanistic target of rapamycin (mTOR) signaling pathway (41). Notably, when the mTOR signaling pathway was inhibited, the inducing effect of IGF-1 was reversed (41). Research by Zhang et al (42) demonstrated that basic FGF and nerve growth factor co-secretion were associated with increased expression levels of phosphorylated (p)-protein kinase B (AKT) and p-extracellular signal-regulated kinases (ERK), thereby suggesting that the ERK and AKT signaling pathway may be involved in the regulation of DPSC neural differentiation. Few investigations into the effects of ET-1 on DPSCs have been conducted; however, researchers have demonstrated that ET-1, secreted by ECs, may promote the interaction of ECs and DPSCs (8). Therefore, the present study aimed to investigate the effects of ET-1 on DPSCs.

The effect of bone dynamic balance and odontogenesis has been a point of interest. Salama et al (43) added ET-1 into the culture medium of MSCs, and reported that ET-1 may modulate the proliferation, migration and differentiation of the MSCs. Research has also suggested that classic wingless-type mouse mammary tumor virus (Wnt) signaling pathways may modulate the differentiation of MSCs into osteoblasts, and modify bone growth via specific gene expression $(44,45)$. Additionally, osteoblasts and osteocytes have been suggested to modulate the formation of osteoclasts via the Wnt/ $\beta$-catenin signaling pathway (46-48). These studies indicated the regulatory effect of the Wnt signaling pathway on bone metabolism. The extracellular molecular mechanism underlying ET-1-mediated bone formation may involve the autocrine or paracrine activation of Wnt signaling that is essential for osteoblast proliferation, differentiation and 
bone development (18). Dickkopf homologue 1 (DDK1) is a selective inhibitor of the Wnt signaling pathway, and its transcription rate may be suppressed by ET-1 in calvarial organ culture. Conversely, recombinant DDK1 may also inhibit ET-1-mediated osteoblast proliferation and new bone formation (18). The present study investigated the promotional effect of cytokines secreted by HUVECs on the differentiation of DPSCs. Furthermore, in order to study the promotional effects of ET-1 on DPSC differentiation, ET-1 was added to the culture medium of DPSCs. The findings of the present study demonstrated that the DPSC + ET-1 group expressed elevated levels of odontogenic markers compared with the DPSC-only group, which indicated the function of ET-1 in the process of DPSC differentiation. However, the function of ET-1 was unclear as in the co-culture group. Since ET-1 may function in an autocrine or paracrine manner, ET-1 exerted a stronger effect on DPSC differentiation compared with in the DPSC-only group, but the mechanisms of action require further investigation. In addition, the alteration of DSP expression in the DPSC + ET-1 group was not as notable as those in the co-culture group, demonstrating that the mechanisms of cytokines are complex. It is possible that two cytokines may synergistically promote differentiation, or inhibit further differentiation following initial promotion. Recently, the cytokines associated with DPSC differentiation have become a focus of research $(49,50)$. The results of the present study suggested that ET-1 may be associated with increases and subsequent decreases in differentiation, which was similar to the trends of the DPSC-only group. Further investigation may be conducted into the maintaining the promotion of differentiation for 21 days.

The small integrin-binding ligand N-linked glycoprotein protein family, including DSP and DMP-1, exhibit positive effects in the process of dentinogenesis $(51,52)$. Dentin sialophosphoprotein (DSPP), a major non-collagenous matrix protein of odontoblasts, undergoes cleavage to dentin phosphoprotein and DSP (53). DSPP is the first putative marker of odontoblastic differentiation and its upregulation has suggested that DPSCs may acquire the capacity to secrete mineralizable dentin (54). DMP-1 is an extracellular matrix glycoprotein important for the mineralization of dentin; during odontoblast maturation, DMP-1 is phosphorylated and exported to the extracellular matrix where it organizes the formation of mineralized matrix $(55,56)$. Research has demonstrated that DMP-1 was initially expressed in mineralized matrix in alveolar bone; however, the expression of DMP-1 was downregulated to lower levels compared with DSP (54). In the present study, the expression levels of DSP were increased compared with DMP-1 at all time points, which was consistent with the aforementioned findings.

In conclusion, HUVECs may promote the differentiation of DPSCs, potentially via a synergetic manner of cytokines secreted by HUVECs. Therefore, DPSCs and ECs may be applicable in regenerative pulp therapy in the future.

\section{Acknowledgements}

The authors are grateful to the Key Laboratory of Myocardial Ischemia of the Ministry of Education (the Second Affiliated Hospital of Harbin Medical University, Harbin, China) for providing facilities to conduct the investigations of the present study.

\section{Funding}

The present study was supported by the Heilongjiang Natural Science Foundation of China under contract no. H2013101, and by the Graduate Science and Technology Innovation Projects program of Harbin Medical University (grant no. YJSCX2016-24HYD).

\section{Availability of data and materials}

The datasets used and/or analyzed during the current study are available from the corresponding author on reasonable request.

\section{Authors' contributions}

ML designed the study, performed the experiments and wrote the manuscript. LZ performed the experiments and organized the images. JH and LW participated in this experiment and made substantial contributions to the acquisition of data. NL analyzed and interpreted the data. DW and XS organized the images and were involved in drafting the manuscript. MY performed the surgery to obtain teeth and was involved in revising the manuscript. WH and XW helped write the manuscript, and were responsible for the study funding and design, as well as providing final approval of the version to be published. All authors read and approved the final manuscript.

\section{Ethics approval and consent to participate}

The present study was approved by the Institutional Ethics Committee of the Second Affiliated Hospital of Harbin Medical University (Harbin, China). Written informed consent was obtained from all patients.

\section{Consent for publication}

Not applicable.

\section{Competing interests}

The authors declare that they have no competing interests.

\section{References}

1. Gronthos S, Mankani M, Brahim J, Robey PG and Shi S: Postnatal human dental pulp stem cells (DPSCs) in vitro and in vivo. Proc Natl Acad Sci USA 97: 13625-13630, 2000.

2. Shi $\mathrm{S}$ and Gronthos S: Perivascular niche of postnatal mesenchymal stem cells in human bone marrow and dental pulp. J Bone Miner Res 18: 696-704, 2003.

3. Spath L, Rotilio V, Alessandrini M, Gambara G, De Angelis L, Mancini M, Mitsiadis TA, Vivarelli E, Naro F, Filippini A and Papaccio G: Explant-derived human dental pulp stem cells enhance differentiation and proliferation potentials. J Cell Mol Med 14: 1635-1644, 2010.

4. Mathieu S, El-Battari A, Dejou J and About I: Role of injured endothelial cells in the recruitment of human pulp cells. Arch Oral Biol 50: 109-113, 2005.

5. Nugent MA and Iozzo RV: Fibroblast growth factor-2. Int J Biochem Cell Biol 32: 115-120, 2000.

6. Dissanayaka WL, Zhan X, Zhang C, Hargreaves KM, Jin L and Tong EH: Coculture of dental pulp stem cells with endothelial cells enhances osteo-/odontogenic and angiogenic potential in vitro. J Endod 38: 454-463, 2012. 
7. Martin P: Wound healing-aiming for perfect skin regeneration Science 276: 75-81, 1997.

8. Wang DS, Miura M, Demura $\mathrm{H}$ and Sato K: Anabolic effects of 1,25-dihydroxyvitamin D3 on osteoblasts are enhanced by vascular endothelial growth factor produced by osteoblasts and by growth factors produced by endothelial cells. Endocrinology 138: 2953-2962, 1997.

9. Saleh FA, Whyte M, Ashton P and Genever PG: Regulation of mesenchymal stem cell activity by endothelial cells. Stem Cells Dev 20: 391-403, 2011.

10. Bouletreau PJ, Warren SM, Spector JA, Peled ZM, Gerrets RP, Greenwald JA and Longaker MT: Hypoxia and VEGF up-regulate BMP-2 mRNA and protein expression in microvascular endothelial cells: Implications for fracture healing. Plast Reconstr Surg 109: 2384-2397, 2002.

11. Liu L, Ling J, Wei X, Wu L and Xiao Y: Stem cell regulatory gene expression in human adult dental pulp and periodontal ligament cells undergoing odontogenic/osteogenic differentiation. J Endod 35: 1368-1376, 2009.

12. Sueyama Y, Kaneko T, Ito T, Kaneko R and Okiji T: Implantation of endothelial cells with mesenchymal stem cells accelerates dental pulp tissue regeneration/healing in pulpotomized rat molars. J Endod 43: 943-948, 2017.

13. Yanagisawa M, Inoue A, Ishikawa T, Kasuya Y, Kimura S, Kumagaye S, Nakajima K, Watanabe TX, Sakakibara S, Goto K, et al: Primary structure, synthesis, and biological activity of rat endothelin, an endothelium-derived vasoconstrictor peptide. Proc Natl Acad Sci USA 85: 6964-6967, 1988.

14. Griswold DE, Douglas SA, Martin LD, Davis TG, Davis L, Ao Z, Luttmann MA, Pullen M, Nambi P, Hay DW and Ohlstein EH: Targeted disruption of the endothelin-B-receptor gene attenuates inflammatory nociception and cutaneous inflammation in mice. J Cardiovasc Pharmacol 36 (5 Suppl 1): S78-S81, 2000.

15. Pomonis JD, Rogers SD, Peters CM, Ghilardi JR and Mantyh PW: Expression and localization of endothelin receptors: Implications for the involvement of peripheral glia in nociception. J Neurosci 21: 999-1006, 2001.

16. Hirata $\mathrm{Y}$ and Ishimaru S: Effects of endothelin receptor antagonists on endothelin-1 and inducible nitric oxide synthase genes in a rat endotoxic shock model. Clin Sci (Lond) 103 (Suppl 48): 332S-335S, 2002.

17. Yanagisawa M, Kurihara H, Kimura S, Tomobe Y, Kobayashi M, Mitsui Y, Yazaki Y, Goto K and Masaki T: A novel potent vasoconstrictor peptide produced by vascular endothelial cells. Nature 332: 411-415, 1988.

18. Sin A, Tang W, Wen CY, Chung SK and Chiu KY: The emerging role of endothelin-1 in the pathogenesis of subchondral bone disturbance and osteoarthritis. Osteoarthritis Cartilage 23: 516-524, 2015.

19. Yuan W, Zhao MD, Yuan FL, Che W, Duan PG, Liu Y and Dong J: Association of endothelin-1 expression and cartilaginous endplate degeneration in humans. PLoS One 8: e60062, 2013.

20. Inoue A, Yanagisawa M, Kimura S, Kasuya Y, Miyauchi T, Goto K and Masaki T: The human endothelin family: Three structurally and pharmacologically distinct isopeptides predicted by three separate genes. Proc Natl Acad Sci USA 86: 2863-2867, 1989.

21. W BF, Kristian HA, Ohlsson L, Tolstrup CA, Warfvinge K and Edvinsson L: Increased endothelin-1-mediated vasoconstriction after organ culture in rat and pig ocular arteries can be suppressed with MEK/ERK1/2 inhibitors. Acta Ophthalmol: Jan 25, 2018 (Epub ahead of print).

22. Rapoport RM and Merkus D: Endothelin-1 regulation of exerciseinduced changes in flow: Dynamic regulation of vascular tone Front Pharmacol 8: 517, 2017.

23. Vanhoutte PM: Say No to ET. J Auton Nerv Syst 81: 271-277, 2000.

24. De Mey JG and Vanhoutte PM: End o' the line revisited: Moving on from nitric oxide to CGRP. Life Sci 118: 120-128, 2014.

25. Shioide M and Noda M: Endothelin modulates osteopontin and osteocalcin messenger ribonucleic acid expression in rat osteoblastic osteosarcoma cells. J Cell Biochem 53: 176-180, 1993.

26. Sasaki T and Hong MH: Localization of endothelin-1 in the osteoclast. J Electron Microsc (Tokyo) 42: 193-196, 1993.

27. Warner TD: Endothelin and its inhibitors. Springer-Verlag, Berlin, pp149-150, 2001.

28. Neuhaus SJ and Byers MR: Endothelin receptors and endothelin-1 in developing rat teeth. Arch Oral Biol 52: 655-662, 2007.
29. Khan ZA, Farhangkhoee H, Mahon JL, Bere L, Gonder JR, Chan BM, Uniyal S and Chakrabarti S: Endothelins: Regulators of extracellular matrix protein production in diabetes. Exp Biol Med (Maywood) 231: 1022-1029, 2006.

30. Gilbert TM, Pashley DH and Anderson RW: Response of pulpal blood flow to intra-arterial infusion of endothelin. J Endod 18: 228-231, 1992.

31. Pourjafar M, Saidijam M, Mansouri K, Malih S, Ranjbar Nejad T, Shabab N and Najafi R: Cytoprotective effects of endothelin-1 on mesenchymal stem cells: An in vitro study. Clin Exp Pharmacol Physiol 43: 769-776, 2016.

32. Liu M, Sun Y, Liu Y, Yuan M, Zhang Z and Hu W: Modulation of the differentiation of dental pulp stem cells by different concentrations of $\beta$-glycerophosphate. Molecules 17: 1219-1232, 2012.

33. Livak KJ and Schmittgen TD: Analysis of relative gene expression data using real-time quantitative PCR and the 2(-Delta Delta C(T)) method. Methods 25: 402-408, 2001.

34. Hutley LJ, Herington AC, Shurety W, Cheung C, Vesey DA, Cameron DP and Prins JB: Human adipose tissue endothelial cells promote preadipocyte proliferation. Am J Physiol Endocrinol Metab 281: E1037-E1044, 2001.

35. VillarsF, Guillotin B,Amédée T,DutoyaS, Bordenave L,Bareille R and Amédée J: Effect of HUVEC on human osteoprogenitor cell differentiation needs heterotypic gap junction communication. Am J Physiol Cell Physiol 282: C775-C785, 2002.

36. Kaigler D, Krebsbach PH, West ER, Horger K, Huang YC and Mooney DJ: Endothelial cell modulation of bone marrow stromal cell osteogenic potential. FASEB J 19: 665-667, 2005.

37. Crisan M, Yap S, Casteilla L, Chen CW, Corselli M, Park TS Andriolo G, Sun B, Zheng B, Zhang L, et al: A perivascular origin for mesenchymal stem cells in multiple human organs. Cell Stem Cell 3: 301-313, 2008.

38. Arai K and Lo EH: An oligovascular niche: Cerebral endothelial cells promote the survival and proliferation of oligodendrocyte precursor cells. J Neurosci 29: 4351-4355, 2009.

39. Vernon SM, Campos MJ, Haystead T, Thompson MM, DiCorleto PE and Owens GK: Endothelial cell-conditioned medium downregulates smooth muscle contractile protein expression. Am J Physiol 272: C582-C891, 1997.

40. Guillotin B, Bareille R, Bourget C, Bordenave L and Amédée J: Interaction between human umbilical vein endothelial cells and human osteoprogenitors triggers pleiotropic effect that may support osteoblastic function. Bone 42: 1080-1091, 2008.

41. Feng X, Huang D, Lu X, Feng G, Xing J, Lu J, Xu K, Xia W, Meng Y, Tao T, et al: Insulin-like growth factor 1 can promote proliferation and osteogenic differentiation of human dental pulp stem cells via mTOR pathway. Dev Growth Differ 56: 615-624, 2014.

42. Zhang J, Lian M, Cao P, Bao G, Xu G, Sun Y, Wang L, Chen J, Wang Y, Feng G and Cui Z: Effects of nerve growth factor and basic fibroblast growth factor promote human dental pulp stem cells to neural differentiation. Neurochem Res 42: 1015-1025, 2017.

43. Salama M, Andrukhova O, Jaksch P, Taghavi S, Kelpetko W, Dekan $\mathrm{G}$ and Aharinejad S: Endothelin-1 governs proliferation and migration of bronchoalveolar lavage-derived lung mesenchymal stem cells in bronchiolitis obliterans syndrome. Transplantation 92: 155-162, 2011.

44. Boland GM, Perkins G, Hall DJ and Tuan RS: Wnt 3a promotes proliferation and suppresses osteogenic differentiation of adult human mesenchymal stem cells. J Cell Biochem 93: 1210-1230, 2004.

45. Liu G, Vijayakumar S, Grumolato L, Arroyave R, Qiao H, Akiri $\mathrm{G}$ and Aaronson SA: Canonical Wnts function as potent regulators of osteogenesis by human mesenchymal stem cells. J Cell Biol 185: 67-75, 2009.

46. Bianco P: Minireview: The stem cell next door: Skeletal and hematopoietic stem cell 'niches' in bone. Endocrinology 152: 2957-2962, 2011.

47. Long F: Building strong bones: Molecular regulation of the osteoblast lineage. Nat Rev Mol Cell Biol 13: 27-38, 2011.

48. Tüysüz B, Bursalı A, AlpZ, Suyugül N, Laine CM and Mäkitie O: Osteoporosis-pseudoglioma syndrome: Three novel mutations in the LRP5 gene and response to bisphosphonate treatment. Horm Res Paediatr 77: 115-120, 2012.

49. Nakayama H, Iohara K, Hayashi Y, Okuwa Y, Kurita K and Nakashima M: Enhanced regeneration potential of mobilized dental pulp stem cells from immature teeth. Oral Dis 23: 620-628, 2017. 
50. He X, Jiang W, Luo Z, Qu T, Wang Z, Liu N, Zhang Y, Cooper PR and He W: IFN- $\gamma$ regulates human dental pulp stem cells behavior via NF-кB and MAPK signaling. Sci Rep 7: 40681, 2017.

51. Batouli S, Miura M, Brahim J, Tsutsui TW, Fisher LW, Gronthos S, Robey PG and Shi S: Comparison of stem-cell-mediated osteogenesis and dentinogenesis. J Dent Res 82: 976-981, 2003.

52. Tye CE, Rattray KR, Warner KJ, Gordon JA, Sodek J, Hunter GK and Goldberg HA: Delineation of the hydroxyapatite-nucleating domains of bone sialoprotein. J Biol Chem 278: 7949-7955, 2003.

53. Qin C, Baba O and Butler WT: Post-translational modifications of sibling proteins and their roles in osteogenesis and dentinogenesis. Crit Rev Oral Biol Med 15: 126-136, 2004.

54. Hao J, Ramachandran A and George A: Temporal and spatial localization of the dentin matrix proteins during dentin biomineralization. J Histochem Cytochem 57: 227-237, 2009.
55. Aplin HM, Hirst KL, Crosby AH and Dixon MJ: Mapping of the human dentin matrix acidic phosphoprotein gene (DMP1) to the dentinogenesis imperfecta type II critical region at chromosome 4q21. Genomics 30: 347-349, 1995.

56. Hirst KL, Simmons D, Feng J, Aplin H, Dixon MJ and MacDougall M: Elucidation of the sequence and the genomic organization of the human dentin matrix acidic phosphoprotein 1 (DMP1) gene: Exclusion of the locus from a causative role in the pathogenesis of dentinogenesis imperfecta type II. Genomics 42: 38-45, 1997.

(i) $\Theta$ This work is licensed under a Creative Common Attribution-NonCommercial-NoDerivatives 4.0 International (CC BY-NC-ND 4.0) License. 